\section{RSP}

http://www.rsp.fsp.usp.br/
Revista de Saúde Pública

\title{
Comportamento sedentário e competência motora em crianças e adolescentes: revisão
}

\author{
Guilherme dos Santos' iD, Paulo Henrique Guerra',"l (D), Suedem Andrade Milani' iD, Ariane \\ Brito Diniz Santos"'I iD, Maria Teresa Cattuzzo"II iD, Alessandro Hervaldo Nicolai Rél iD \\ ' Universidade de São Paulo. Escola de Artes, Ciências e Humanidades. Programa de Pós-Graduação em \\ Ciências da Atividade Física. São Paulo, SP, Brasil \\ " Universidade Federal da Fronteira Sul. Chapecó, SC, Brasil \\ III Universidade de Pernambuco. Escola Superior de Educação Física. Recife, PE, Brasil
}

\author{
Correspondência: \\ Guilherme dos Santos \\ Rua Arlindo Béttio, 1000 - Ermelino \\ Matarazzo \\ 03828-000 São Paulo, SP, Brasil \\ E-mail: guilherme4.santos@usp.br \\ Recebido: 16 jul 2020 \\ Aprovado: 17 nov 2020 \\ Como citar: Santos G, Guerra \\ $\mathrm{PH}$, Milani SA, Santos ABD, \\ Cattuzzo MT, Ré AHN. \\ Comportamento sedentário e \\ competência motora em crianças \\ e adolescentes: revisão. Rev \\ Saude Publica. 2021;55:57. \\ https://doi.org/10.11606/s1518- \\ 8787.2021055002917
}

Copyright: Este é um artigo de acesso aberto distribuído sob os termos da Licença de Atribuição Creative Commons, que permite uso irrestrito, distribuição e reprodução em qualquer meio, desde que o autor e a fonte originais sejam creditados.

\section{RESUMO}

OBJETIVO: Sintetizar as evidências de estudos que analisaram as associações entre comportamento sedentário e competência motora em crianças e adolescentes.

MÉTODOS: Revisão sistemática de artigos originais que analisaram possíveis associações entre comportamento sedentário e competência motora em crianças e adolescentes (3-18 anos de idade), sem restrições quanto ao delineamento de estudo, instrumentos e protocolos de análise. Os artigos foram identificados por meio de buscas nas bases PubMed, Web of Science, Academic Search Premier, Cinahl, Medline e SPORTDiscus, assim como em listas de referências. O nível de evidência foi avaliado de acordo com a quantidade de estudos que reportaram significância estatística nas associações entre as variáveis e a qualidade dos artigos (o risco de viés).

RESULTADOS: De 2.462 estudos iniciais, 22 compuseram a síntese (duas intervenções, nove longitudinais e onze transversais). Desses, em 13 foram observadas associações negativas entre as variáveis, mais frequentemente na faixa etária de sete a catorze anos. Na análise do risco de viés, as principais limitações dos estudos foram "amostragens por conveniência" e "não descrição do dimensionamento amostral".

CONCLUSÕES: A evidência disponível sugere que o comportamento sedentário está negativamente associado à competência motora em crianças do ensino fundamental, embora a evidência seja incerta nos anos pré-escolares; a síntese de resultados dos estudos longitudinais sugere que o comportamento sedentário afeta negativamente o desenvolvimento da competência motora. É importante que futuros estudos tenham maior controle sobre os determinantes socioculturais e aprofundem os conhecimentos em relação ao sexo e à faixa etária, assim como dos métodos e indicadores utilizados para avaliação das duas variáveis.

DESCRITORES: Criança. Adolescente. Comportamento Sedentário. Competência Motora. Revisão Sistemática. 


\section{INTRODUÇÃO}

O elevado índice de comportamento sedentário (CS), definido por atividades com gasto energético $\leq 1,5$ equivalentes metabólicos (MET) enquanto sentado ou deitado durante o período de vigília ${ }^{1}$, é atualmente um grave problema de saúde pública mundial ${ }^{2,3}$. O CS durante a infância e adolescência tem sido identificado como um preditor do CS na idade adulta ${ }^{4,5}$ e desenvolvimento de doenças crônicas como diabetes, hipertensão e sobrepeso ou obesidade ${ }^{6-9}$.

Diversos fatores, como o acesso às tecnologias, a diminuição da oferta de espaço público e os índices de violência, têm levado crianças e adolescentes a adotar um CS durante grande parte de seu dia ${ }^{10-12}$, particularmente em opções de lazer que envolvem atividades de tela (tablets, smartphones, computadores, videogames, televisão) ${ }^{10,13,14}$. Diretrizes atuais sugerem um limite diário de $1 \mathrm{~h}$ em atividades de tela para a faixa etária de 3 a 5 anos e de $2 \mathrm{~h}$ diárias dos 5 aos 17 anos $^{15,16}$. Apesar dessas recomendações, têm sido reportado valores elevados de tempo de tela (mais de 3 a 4 h por dia) em crianças e adolescentes de diferentes nacionalidades ${ }^{10,17,18}$, fato provavelmente agravado em função da pandemia de covid-19 ${ }^{19}$.

Juntamente com os altos índices de CS, também tem sido reportado um declínio na competência motora $(\mathrm{CM})^{20-22}$, ou seja, competência na execução de habilidades motoras, com organização e controle de movimentos compatível com a idade ${ }^{23}$; esse fator é potencialmente importante para a diminuição nos índices de CS e aumento da prática de atividade física $(\mathrm{AF})^{12,24}$. Um crescente corpo de evidências tem indicado que a CM favorece a participação em atividades físicas e esportivas ${ }^{25-27}$ e está associada a melhores resultados gerais de saúde, incluindo um peso corporal adequado e maior aptidão cardiorrespiratória ${ }^{28}$. Crianças e adolescentes com baixa CM tendem a evitar a prática de atividade física ${ }^{12,17}$ e adotar atividades de tela como opção de lazer ${ }^{17,29}$, o que restringe ainda mais o desenvolvimento motor e pode gerar um ciclo comportamental negativo, aumentando a probabilidade de inatividade física e CS excessivo ao longo da vida. Pesquisas recentes têm encontrado uma associação negativa entre $\mathrm{CS}$ e $\mathrm{CM}^{12,30}$ que sugere uma relação recíproca entre essas variáveis ${ }^{24}$, com importante aplicação em políticas de promoção da saúde.

Entretanto, em artigo de metanálise, a evidência de associação entre CS e CM foi considerada incerta ${ }^{31}$ pois, além de terem sido identificadas apenas três pesquisas com crianças e adolescentes ${ }^{32-34}$, em somente uma delas ${ }^{33}$, realizada com crianças de 9 a 10 anos de idade, houve associação significante. Portanto, em função do possível impacto das diretrizes de CS em políticas de saúde ${ }^{15}$ e considerando a importância do desenvolvimento da CM na juventude ${ }^{23,24,28}$, existe a necessidade de um maior entendimento da associação entre CM e CS, levando em conta um maior número de pesquisas e as possíveis diferenças entre faixas etárias.

Assim, o objetivo desta revisão sistemática foi sintetizar as evidências de estudos que analisaram as associações entre CS e CM em crianças e adolescentes.

\section{MÉTODOS}

\section{Protocolo e Registro}

O presente estudo configura-se como uma revisão sistemática, tendo seu protocolo registrado no International Prospective Register of Systematic Reviews (PROSPERO CRD42020161554). O texto integral foi elaborado com base nos itens da lista Preferred Reporting Items of Systematic Reviews and Meta-Analyses (PRISMA) ${ }^{35}$.

\section{Critérios de Elegibilidade}

Com base na questão de pesquisa, foram procurados artigos originais publicados em revistas científicas com avaliação por pares nos idiomas inglês, português e 
espanhol. Mais especificamente, outros itens foram estabelecidos a partir da estratégia "Picos" ${ }^{36}$, considerando:

\section{População}

Amostras heterogêneas de crianças e adolescentes (sem deficiências nem quadros clínicos específicos, à exceção de amostras especificamente compostas por crianças com sobrepeso ou obesidade) com idade entre 3 e 18 anos. Para nossos fins, visando melhorar o grau de comparabilidade e apresentação da evidência, os subgrupos foram definidos da seguinte forma, considerando o sistema de educação brasileiro: pré-escolares, dos 3 aos 6 anos de idade; ensino fundamental, envolvendo crianças e adolescentes entre 6 e 14 anos; e ensino médio, que abrange adolescentes entre 15 e 18 anos.

\section{Intervenção ou Exposição}

Foram incluídos estudos de intervenção que implementaram estratégias para o controle ou redução do CS, independentemente do contexto (por exemplo, na escola ou na comunidade) e características (seja por atividades teóricas, práticas ou ambas).

Já os estudos observacionais analisaram possíveis associações entre o CS e a CM, tomando como base o CS enquanto variável de exposição e a CM enquanto variável de desfecho. Para constar, não foram impostas restrições relacionadas aos tipos (ex.: tempo de tela, tempo sentado), domínios (ex.: lazer, escola e deslocamento) e instrumentos (ex.: questionários e sensores de movimento) utilizados para a mensuração do CS, assim como para a CM. Considerando o atual entendimento do conceito, foram excluídos os estudos que abordaram "sedentário" enquanto ausência de atividade física.

\section{Comparação}

Nos estudos de intervenção, não foram impostas restrições quanto à existência ou tipo de atividades oferecidas aos grupos controle, optando-se, quando possível (como em estudos com mais de um grupo controle), por comparadores que receberam menor carga de atividade.

\section{Desfechos (Indicadores de Saúde)}

O desfecho foi a competência motora. Para avaliá-la, foram consideradas as habilidades motoras grossas de locomoção, controle de objetos e equilíbrio.

\section{Delineamento do Estudo}

Foram incluídos estudos transversais, coortes e intervenções que apresentassem análises sobre possíveis associações entre o CS e a CM, independentemente do protocolo utilizado (ex.: análises univariadas ou multivariadas). Estudos de caso, descritivos, revisões, metanálises, dissertações, teses e resumos de eventos foram excluídos.

\section{Fontes de Informação e Estratégia de Busca}

Para recuperação dos estudos potenciais, em março de 2020, buscas sistemáticas foram aplicadas em seis bases de dados eletrônicas: PubMed, Web of Science, Academic Search Premier, Cinahl, Medline e SPORTDiscus, partindo da estratégia aplicada no PubMed: $(((()(((($ motor competence[Text Word]) OR motor development[Text Word]) OR gross motor skills[Text Word]) OR fundamental motor skills[Text Word]) OR fundamental movement skills[Text Word]) OR motor coordination[Text Word]) OR motor ability[Text Word]) OR locomotor skills[Text Word]) OR object control skills[Text Word]) OR motor skills[Text Word]) AND ((((((sedentary behavior[Text Word]) OR sitting time[Text Word]) OR television[Text Word]) OR computer[Text Word]) OR videogame[Text Word]) OR screen time[Text Word]) OR screen activit*[Text Word]) AND child*[Text Word]. Para evitar perda de informação relevante, buscas manuais foram conduzidas nas listas de referências dos 
artigos avaliados pelos seus textos integrais. Não foram impostas restrições em relação ao ano de publicação.

Um autor realizou a busca inicial e introduziu todos os artigos recuperados na plataforma Rayyan (https://rayyan.qcri.org), onde foi conduzida identificação e remoção das duplicatas interbases de dados. Dois autores (GS e SAM), de forma independente, revisaram os artigos disponíveis pelos títulos e resumos. Os resultados foram comparados e as inconsistências foram discutidas até se alcançar um consenso. Caso não se chegasse ao consenso, um terceiro autor (AHNR) definiria a elegibilidade do estudo. Terminada essa fase, os mesmos autores avaliaram os textos completos dos artigos remanescentes.

\section{Extração de Dados}

Os dados foram extraídos de forma independente por dois autores (GS e SAM), utilizando uma planilha eletrônica, que foi organizada em dois níveis de informações: (1) descritivas (local, desenho, amostra e idade) e (2) metodológicas (tipo e medida de CM, tipo e medida de CS, estatística e principais resultados). Em particular, foram extraídos os resultados relacionados às análises entre $\mathrm{CS}$ e $\mathrm{CM}$, considerando as associações positivas, negativas ou nulas, de acordo com magnitude e nível de significância $p<0,05$. Os dados foram extraídos independentemente por sexo apenas se os dados da amostra total não estivessem disponíveis. No caso de resultados diferentes para cada sexo, o estudo foi classificado como de associação incerta.

\section{Risco de Viés e Avaliação da Qualidade dos Estudos}

Todos os estudos incluídos tiveram seu risco de viés avaliado por dois autores (GS e SAM), de forma independente, com apoio do pesquisador sênior (AHNR). Para tanto, foi utilizado o instrumento desenvolvido por Lubans et al. ${ }^{27}$, que, por sua vez, basearam-se nos itens das diretrizes STROBE e CONSORT. Foram atribuídas pontuações de 0 (ausente ou inadequadamente descrito) ou 1 (presente e descrito adequadamente) em seis questões, a saber: (a) "O estudo descreve os critérios de elegibilidade/seleção dos participantes?"; (b) “Os participantes foram selecionados aleatoriamente?”; (c) “O estudo menciona fontes e detalhes da avaliação da CM, e esses instrumentos têm confiabilidade adequada para essa faixa etária específica?”; (d) “O estudo menciona fontes e detalhes da avaliação de CS, e todos os métodos têm confiabilidade aceitável?”; (e) O estudo relatou dimensionamento amostral e foi adequadamente dimensionado para detectar relações hipotéticas?”; (f) "O estudo menciona o número de sujeitos que completaram cada uma das diferentes medidas, e esses participantes concluíram pelo menos $80 \%$ das medidas de CM e CS?". Estabeleceu-se previamente que estudos com pontuação $\leq 2$ teriam alto risco de viés; estudos na faixa entre 3 e 4 pontos, médio risco de viés; e estudos com pontuação entre 5 e 6, baixo risco de viés.

\section{Síntese de Resultados}

Considerando a heterogeneidade entre os delineamentos e os métodos adotados, desde as primeiras tratativas, estipulou-se a construção de uma síntese descritiva dos resultados disponíveis. O julgamento da evidência científica foi baseado em Lubans et al. ${ }^{27}$, usando a porcentagem de estudos que reportaram significância estatística na associação, mas também levando em consideração o risco de viés: (a) falta de evidência científica, se menos de $33 \%$ dos estudos indicarem associação significativa entre as variáveis ou nenhum dos estudos considerados de baixo risco de viés encontrarem uma associação significativa; (b) evidência incerta, se 34 a 59\% dos estudos indicarem uma associação significativa entre as variáveis; (c) evidência positiva (ou negativa), se 60 a 100\% dos estudos indicarem uma significativa associação entre variáveis; (d) forte evidência, se 60 a 100\% dos estudos indicarem uma associação significativa entre variáveis (na mesma direção), não houver estudos classificados como associação incerta e mais de 59\% dos estudos forem considerados de baixo risco de viés (pontuação $\geq 5$ ). 


\section{RESULTADOS}

O fluxograma apresenta o processo de seleção (Figura 1). De forma resumida, das 2.462 referências inicialmente identificadas, 1.336 foram avaliadas pelos títulos e resumos. Dessas, 36 foram encaminhadas para triagem por leitura integral dos textos e 14 foram excluídas por não apresentar indicadores de CS $(\mathrm{n}=12)$ ou não incluir a faixa etária que era alvo do presente estudo $(\mathrm{n}=2)$. Finalmente, 22 estudos foram incluídos na revisão sistemática ${ }^{12,17,30,32,33,37-53}$.

A metade dos estudos incluídos apresentou desenho transversal $(n=11)$, destacando-se também a presença de nove estudos longitudinais e dois experimentais (Tabela 1). As amostras investigadas incluíam pessoas de 3 a 14 anos de idade. O tamanho da amostra variou de $17^{49}$ a $10.831^{52}$ participantes. A maior parte dos estudos foi conduzida nos países da América do Norte $(\mathrm{n}=9)^{17,33,37,38,40,43-45,53}$.

Em relação às medidas, catorze estudos usaram medidas de produto - como BruininksOseretsky Test of Motor Proficiency Second Edition (BOT-2), Körperkoordinationstest für Kinder (KTK) e Movement Assessment Battery for Children Second Edition (MABC-2) para avaliar a CM, sete utilizaram medidas de processo - como o Test of Gross Motor Development (TGMD) - e apenas um estudo ${ }^{17}$ utilizou ambos os tipos. O TGMD (primeira, segunda ou terceira versão) foi o teste motor mais utilizado $(n=8)^{17,38-42,49,53}$. Três estudos utilizaram o PE Metrics ${ }^{43-45}$, três estudos utilizaram o BOT-2 ${ }^{37,40,51}$, dois estudos utilizaram o KTK ${ }^{33,48}$ e dois estudos utilizaram o MABC-2 ${ }^{17,50}$. Os testes Athletic Skills Track ${ }^{12}$ e Deutsche Motorik Test ${ }^{30}$, foram utilizados em um estudo. Três estudos utilizaram tarefas motoras específicas (ex.: andar para trás, equilíbrio, arremessar e agarrar) ${ }^{46,47,52}$. Com relação à medida de CS, dezesseis estudos utilizaram acelerômetro como medida direta e seis estudos fizeram uso de questionários. Com relação aos questionários, cinco estudos os utilizaram para avaliar tempo de tela recreativo e apenas um estudo os utilizou para avaliar tempo sentado (Tabela 2).

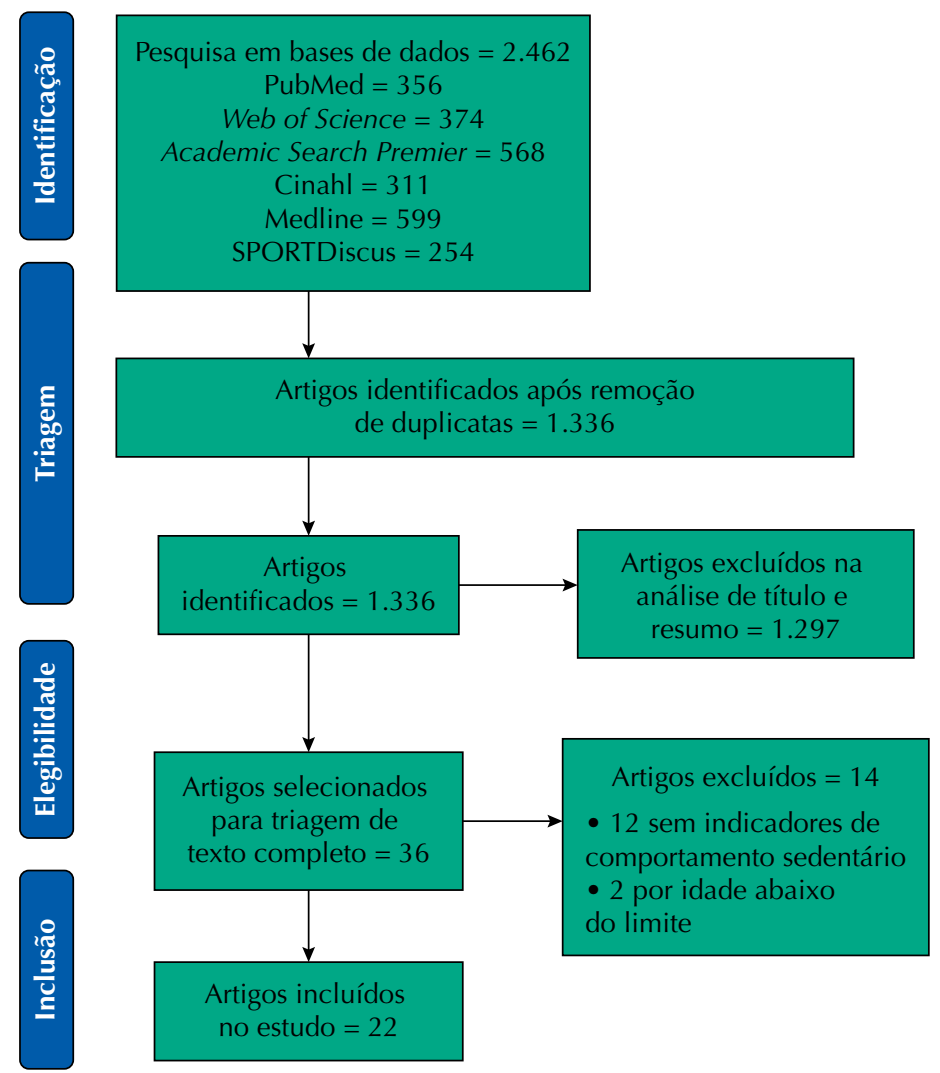

Figura 1. Fluxograma da revisão sistemática. 
Tabela 1. Característica descritiva dos estudos incluídos.

\begin{tabular}{|c|c|c|c|c|}
\hline Estudo & Local & Delineamento & Amostra & Idade \\
\hline Adank et al. ${ }^{12}, 2018$ & Holanda & Transversal & 595 (291 meninos) & $7-11$ anos \\
\hline Barnett et al. ${ }^{39}, 2012$ & Austrália & Transversal & 53 (22 meninos) & $3-6$ anos \\
\hline Burns et al. ${ }^{38}, 2019$ & Estados Unidos & Transversal & 409 (205 meninos) & $\begin{array}{c}1^{\circ} \text { ao } 5^{\circ} \text { ano; média de } \\
8,4 \text { anos (DP }=1,8)\end{array}$ \\
\hline Cadoret et al. $.^{40}, 2018$ & Canadá & Longitudinal & 133 (51 meninos) & 4-7 anos \\
\hline Capio et al. ${ }^{41}, 2015$ & Hong Kong & Experimental & 26 (13 meninos) & $\begin{array}{l}\text { Grupo experimental: } \\
\text { média de } 7,17 \text { anos (DP } \\
=2,77 \text { ); grupo controle: } \\
\text { média de } 6,82 \text { anos (DP } \\
=2,51 \text { ) }\end{array}$ \\
\hline Cliff et al. ${ }^{32}, 2009$ & Austrália & Transversal & 46 (25 meninos) & $3-5$ anos \\
\hline Drenowatz e Greierº, 2019 & Áustria & Longitudinal & 213 (122 meninos) & $\begin{array}{l}5^{\circ} \text { ano; média de } 10,4 \\
\text { anos }(\mathrm{DP}=0,6)\end{array}$ \\
\hline Famelia et al. ${ }^{42}, 2017$ & Indonésia & Transversal & 66 (30 meninos) & $3-6$ anos \\
\hline $\mathrm{Gu}^{43}, 2016$ & Estados Unidos & Longitudinal & 256 (129 meninos) & $5-6$ anos \\
\hline Gu et al. ${ }^{44}, 2018$ & Estados Unidos & Longitudinal & 141 (72 meninos) & $\begin{array}{l}\text { Pré-escolares; média de } \\
5,37 \text { anos }(\mathrm{DP}=0,48)\end{array}$ \\
\hline Gu, Chen e Zhang 45,2019 & Estados Unidos & Longitudinal & 671 (363 meninos) & $\begin{array}{l}\text { Média de } 6,96 \text { anos } \\
\quad(\mathrm{DP}=1,60)\end{array}$ \\
\hline Jaakkola et al. ${ }^{46}, 2009$ & Finlândia & Transversal & 152 (76 meninos) & $7^{\circ}$ ano; 13 anos \\
\hline Jaakkola et al. ${ }^{47}, 2019$ & Finlândia & Longitudinal & 336 (173 meninos) & $\begin{array}{c}6^{\circ} \text { ano; média de } 12,02 \\
\text { anos }(\mathrm{DP}=0,38)\end{array}$ \\
\hline Laukkanen et al. ${ }^{48}, 2014$ & Finlândia & Experimental & 84 (38 meninos) & $5-8$ anos \\
\hline Lloyd et al. ${ }^{49}, 2014$ & Canadá & Longitudinal & 17 (5 meninos) & 6 anos \\
\hline Lopes et al. ${ }^{33}, 2012$ & Portugal & Transversal & 213 (103 meninos) & 9-10 anos \\
\hline Lopes et al. ${ }^{50}, 2016$ & Portugal & Transversal & 101 (53 meninos) & $3-5$ anos \\
\hline Matarma et al. ${ }^{51}, 2018$ & Finlândia & Longitudinal & 111 (45 meninos) & $5-6$ anos \\
\hline Smith et al. ${ }^{52}, 2015$ & Inglaterra & Longitudinal & $10831(\mathrm{~N} / \mathrm{A})$ & 10 anos \\
\hline Tsuda et al. ${ }^{53}, 2019$ & Estados Unidos & Transversal & 72 (39 meninos) & $\begin{array}{c}\text { Pré-escolares; média de } \\
4,38 \text { anos }(\mathrm{DP}=0,85)\end{array}$ \\
\hline Webster et al. ${ }^{17}, 2019$ & Estados Unidos & Transversal & 126 (58 meninos) & $3-4$ anos \\
\hline Wrotniak et al. ${ }^{37}, 2006$ & Estados Unidos & Transversal & 65 (31 meninos) & 8-10 anos \\
\hline
\end{tabular}

DP: desvio-padrão.

Quanto ao risco de viés, $18,2 \%(\mathrm{n}=4)$ dos estudos obtiveram uma pontuação de baixo risco $(\geq 5), 77,3 \%(n=17)$ obtiveram uma pontuação de médio risco e apenas um estudo obteve uma pontuação de alto risco. Todos os estudos atingiram os critérios: (a) "O estudo descreve os critérios de elegibilidade/seleção dos participantes?” e (d) "O estudo menciona fontes e detalhes da avaliação do CS?”. Finalmente, os itens de qualidade que estiveram mais ausentes foram: (b) "Os participantes foram selecionados aleatoriamente?" e (e) "O estudo relatou dimensionamento amostral e foi adequadamente dimensionado para detectar relações hipotéticas?” (Figura 2).

Do total de estudos investigados $(\mathrm{n}=22)$, treze apontaram associações negativas entre CS e CM, seis não apontaram associação e três apontaram associações incertas.

No estrato de estudos avaliados com baixo risco de viés $(n=4)$, foi encontrada associação negativa em três estudos, destacando como principais resultados: CS e alta CM em escolares $^{12}$; CS e CM em pré-escolares ${ }^{44}$; CM na infância e tempo sentado após 20 anos ${ }^{49}$. Resultados de associação negativa também foram encontrados em estudos com risco de viés moderado $30,33,37,40,41,43,45,46,52,53$.

Considerando os estudos experimentais $(\mathrm{n}=2)$ de médio risco de viés, um estudo ${ }^{41}$ encontrou associação negativa entre CS e CM nas habilidades de locomoção e controle de objetos, porém 
Tabela 2. Característica metodológica das variáveis CM e CS, análise estatística e principais resultados.

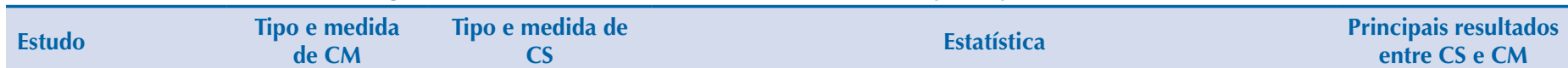

Análise multivariada

Adank et al. ${ }^{12}$

2018

Produto; AST

Tempo total em CS; acelerômetro

Barnett et al. ${ }^{39}$,

Processo;

2012

TGMD-2

Tempo de tela

recreativo;

questionário

Burns et al. ${ }^{38}, 2019 \quad$ Processo;

TGMD-3

Tempo total em CS; acelerômetro

Produto;
BOT-2 SF

Cadoret et al. ${ }^{40}$,

2018

questionário

Tempo total em CS;

2015*

TGMD-2

acelerômetro

Muito baixa CM e CS: $\beta=3,17$; IC 1,28-5,05 entre CS e CM

\begin{tabular}{|c|c|c|}
\hline Cliff et al. ${ }^{32}, 2009$ & $\begin{array}{l}\text { Processo; } \\
\text { TGMD-2 }\end{array}$ & $\begin{array}{c}\text { Tempo total em C } \\
\text { acelerômetro }\end{array}$ \\
\hline $\begin{array}{l}\text { Drenowatz e } \\
\text { Greier }^{30}, 2019\end{array}$ & $\begin{array}{l}\text { Produto; } \\
\text { DMT 6-18 }\end{array}$ & $\begin{array}{l}\text { Tempo de tela } \\
\text { recreativo; } \\
\text { questionário }\end{array}$ \\
\hline $\begin{array}{l}\text { Famelia et al. }{ }^{42} \text {, } \\
2017\end{array}$ & $\begin{array}{l}\text { Processo; } \\
\text { TGMD-3 }\end{array}$ & $\begin{array}{c}\text { Tempo total em C } \\
\text { acelerômetro }\end{array}$ \\
\hline $\mathrm{Gu}^{43}, 2016$ & $\begin{array}{l}\text { Produto; } \\
\text { PE Metrics }\end{array}$ & $\begin{array}{c}\text { Tempo total em C } \\
\text { acelerômetro }\end{array}$ \\
\hline Gu et al. ${ }^{44}, 2018$ & $\begin{array}{l}\text { Produto; } \\
\text { PE Metrics }\end{array}$ & $\begin{array}{l}\text { Tempo total em C } \\
\text { acelerômetro }\end{array}$ \\
\hline $\begin{array}{l}\text { Gu, Chen e } \\
\text { Zhang }{ }^{45}, 2019\end{array}$ & $\begin{array}{l}\text { Produto; } \\
\text { PE Metrics }\end{array}$ & $\begin{array}{l}\text { Tempo total em C } \\
\text { acelerômetro }\end{array}$ \\
\hline $\begin{array}{l}\text { Jaakkola et al. }{ }^{46} \text {, } \\
2009\end{array}$ & $\begin{array}{c}\text { Produto; } \\
\text { Arremesso, salto } \\
\text { e equilíbrio }\end{array}$ & $\begin{array}{c}\text { Tempo de tela } \\
\text { recreativo; } \\
\text { questionário }\end{array}$ \\
\hline
\end{tabular}

Jaakkola et al. ${ }^{47}$,

2019

Produto; Cinco

saltos, arremesso

e agarrar

Laukkanen et al. ${ }^{48}, \quad \begin{aligned} & \text { Produto; } \\ & 2014 \\ & \text { KTK e TCB }\end{aligned}$

Lloyd et al. ${ }^{49}, 2014$ Processo; TGMD
Tempo total em CS; acelerômetro

Tempo total em CS; acelerômetro

Tempo sentado; questionário
Baixa CM e CS: $\beta=1,97$; IC 0,44-3,49

Alta CM e CS: $\beta=-0,45$; IC $-1,71-0,81$

Muito alta CM e CS: $\beta=-1,72$; IC $-3,18-0,27$

Regressão linear hierárquica, ajustada por idade e sexo

Habilidades de controle de objeto e CS: $\beta=-0,13$

Habilidades de locomoção e CS: $\beta=-0,24$

Modelos lineares gerais de efeitos mistos ajustados à idade,

índice de massa corporal, capacidade aeróbica estimada e escola

Habilidades de locomoção e CS: $\gamma=-9,07$

Habilidades de controle de objetos e CS: $\gamma=2,09$

$$
\text { CM e CS: } \gamma=5,05
$$

Correlação de Pearson

CS aos 4 anos e CM aos 7 anos: $r=-0,267$

CS aos 5 anos e CM aos 7 anos: $r=-0,268$

CS aos 7 anos e CM aos 7 anos: $r=-0,246$

Correlação de Pearson

Habilidades de locomoção e CS: $r=-0,310$

Habilidades de controle de objetos e CS: $r=-0,275$

Duração da corrida e CS: $r=0,603$

Distância do salto e CS: $r=-0,445$

Chute e CS: $r=-0,411$

Arremesso e CS: $r=0,328$

Agarrar e CS: $r=-0,242$
Correlação de Pearson; CM e CS em meninos: $r=-0,194$ CM e CS em meninas: $r=0,138$

Manova e correlação de Pearson

Maior consumo de mídia com melhorias nos saltos laterais

$(p=0,03)$ e diminuição na corrida de 6 minutos $(p=0,03)$

Regressão múltipla

Habilidades de locomoção e CS durante o playground: $r=-0,56$ Associação incerta

Habilidades com bola e CS durante o playground: $r=-0,14$

\section{Correlação de Pearson}

Habilidades de locomoção e CS: $r=-0,13$

Habilidades de controle de objetos e CS: $r=-0,16$

CM e CS: $r=-0,19$

Correlação de Pearson

Habilidades de locomoção e CS: $r=-0,30$

Habilidades de controle de objetos e CS: $r=-0,30$

$$
\text { CM e CS: } r=-0,34
$$

Associação negativa

Associação nula

Associação nula

Associação negativa

Associação negativa

Correlação de Pearson

Habilidades de locomoção e CS em hispânicos: $r=-0,25$

Habilidades de controle de objetos e CS em hispânicos:

$$
r=-0,08
$$

Associação negativa

Habilidades de locomoção e CS em não hispânicos: $r=-0,16$

Habilidades de controle de objetos e CS em não hispânicos:

$$
r=-0,06
$$

Correlação de Pearson

Arremesso e CS: $r=0,09$

Salto e CS: $r=-0,28$

Equilíbrio e CS: $r=0,22$

Associação negativa

Modelagem de equações estruturais

$6^{\circ}$ ano: Habilidades de locomoção e CS: $r=-0,056$

Habilidades de controle de objetos e CS: $r=-0,142$

$7^{\circ}$ ano: Habilidades de locomoção e CS: $r=-0,364$

Habilidades de controle de objetos e CS: $r=-0,059$

$$
\text { Correlação parcial }
$$

CM e CS em pré-escolares meninos: $r=-0,52$

Correlação de Pearson

Habilidades de locomoção e CS: $r=-0,37$

Habilidades de controle de objetos e CS: $-0,10$

$$
\text { CM e CS: } r=-0,25
$$

Associação negativa 
Tabela 2. Característica metodológica das variáveis CM e CS, análise estatística e principais resultados. Continuação

\begin{tabular}{|c|c|c|c|c|}
\hline Lopes et al. ${ }^{33}, 2012$ & Produto; KTK & $\begin{array}{l}\text { Tempo total em CS; } \\
\text { acelerômetro }\end{array}$ & $\begin{array}{c}\text { Regressão logística binária } \\
R P=5,065 \text { para meninas e } R P=9,149 \text { para meninos }\end{array}$ & Associação negativa \\
\hline Lopes et al. ${ }^{50}, 2016$ & $\begin{array}{l}\text { Produto; MABC- } \\
\qquad 2\end{array}$ & $\begin{array}{l}\text { Tempo total em CS; } \\
\text { acelerômetro }\end{array}$ & $\begin{array}{c}\text { Correlação de Spearman } \\
\text { Equilíbrio e CS: } r=0,15 \\
\text { Habilidades de controle de objetos e CS: } r=0,03\end{array}$ & Associação nula \\
\hline $\begin{array}{l}\text { Matarma et al. }{ }^{51}, \\
2018\end{array}$ & Produto; BOT-2 & $\begin{array}{l}\text { Tempo total em CS; } \\
\text { acelerômetro }\end{array}$ & $\begin{array}{l}\text { Regressão linear } \\
\text { Sem correlação significativa }\end{array}$ & Associação nula \\
\hline Smith et al. ${ }^{52}, 2015$ & $\begin{array}{l}\text { Produto; } \\
\text { arremesso, } \\
\text { equilibrar em um } \\
\text { pé, andar para } \\
\text { trás }\end{array}$ & $\begin{array}{l}\text { Tempo de tela } \\
\text { recreativo; } \\
\text { questionário }\end{array}$ & $\begin{array}{c}\text { Regressão logística } \\
\text { Alta CM e baixa probabilidade de alto tempo de tela aos } \\
16 \text { anos: } \mathrm{RP}=0,79 ; \mathrm{IC} 0,64-0,98 \\
\text { Alta CM e baixa probabilidade de tempo de TV aos } 42 \text { anos: } \\
\mathrm{RP}=0,85 ; \mathrm{IC} 0,72-0,99\end{array}$ & Associação negativa \\
\hline Tsuda et al. ${ }^{53}, 2019$ & $\begin{array}{l}\text { Processo; } \\
\text { TGMD-2 }\end{array}$ & $\begin{array}{l}\text { Tempo total em CS; } \\
\text { acelerômetro }\end{array}$ & $\begin{array}{c}\text { Correlação de Pearson } \\
\text { Habilidades de locomoção e CS: } r=-0,46 \\
\text { Habilidades de controle de objetos e CS: } r=-0,42\end{array}$ & Associação negativa \\
\hline $\begin{array}{l}\text { Webster et al. }{ }^{17} \text {, } \\
2019\end{array}$ & $\begin{array}{l}\text { Processo e } \\
\text { produto; TGMD- } \\
3 \text { e MABC-2 }\end{array}$ & $\begin{array}{l}\text { Tempo total em CS; } \\
\text { acelerômetro }\end{array}$ & $\begin{array}{l}\text { Correlação de Pearson } \\
\text { CM e tempo de tela: } \beta=-1,6\end{array}$ & Associação nula \\
\hline $\begin{array}{l}\text { Wrotniak et al. }{ }^{37}, \\
2006\end{array}$ & $\begin{array}{l}\text { Produto; } \\
\text { BOTMP-SF }\end{array}$ & $\begin{array}{l}\text { Tempo total em CS; } \\
\text { acelerômetro }\end{array}$ & $\begin{array}{l}\text { Correlação de Pearson } \\
\text { CM e CS: } r=-0,308\end{array}$ & Associação negativa \\
\hline
\end{tabular}

CM: competência motora; CS: comportamento sedentário; RP: razão de probabilidade; IC: intervalo de confiança; Manova: análise multivariada da variância; AST: Athletic Skills Track; BOT-2: Bruininks-Oseretsky Test of Motor Proficiency Second Edition; BOT-2 SF: Bruininks-Oseretsky Test of Motor Proficiency Second Edition - Short Form; BOTMP-SF: Bruininks-Oseretsky Test of Motor Proficiency - Short Form; DMT 6-18: Deutsche Motorik Test; KTK: Körperkoordinationstest für Kinder; MABC-2: Movement Assessment Battery for Children Second Edition; TCB: Underarmor Throw and Catch a Ball; TGMD: Test of Gross Motor Development [segunda e terceira edições assinaladas pelo número após a sigla].

Nota: Apenas os dados de crianças com desenvolvimento típico foram considerados para os resultados.

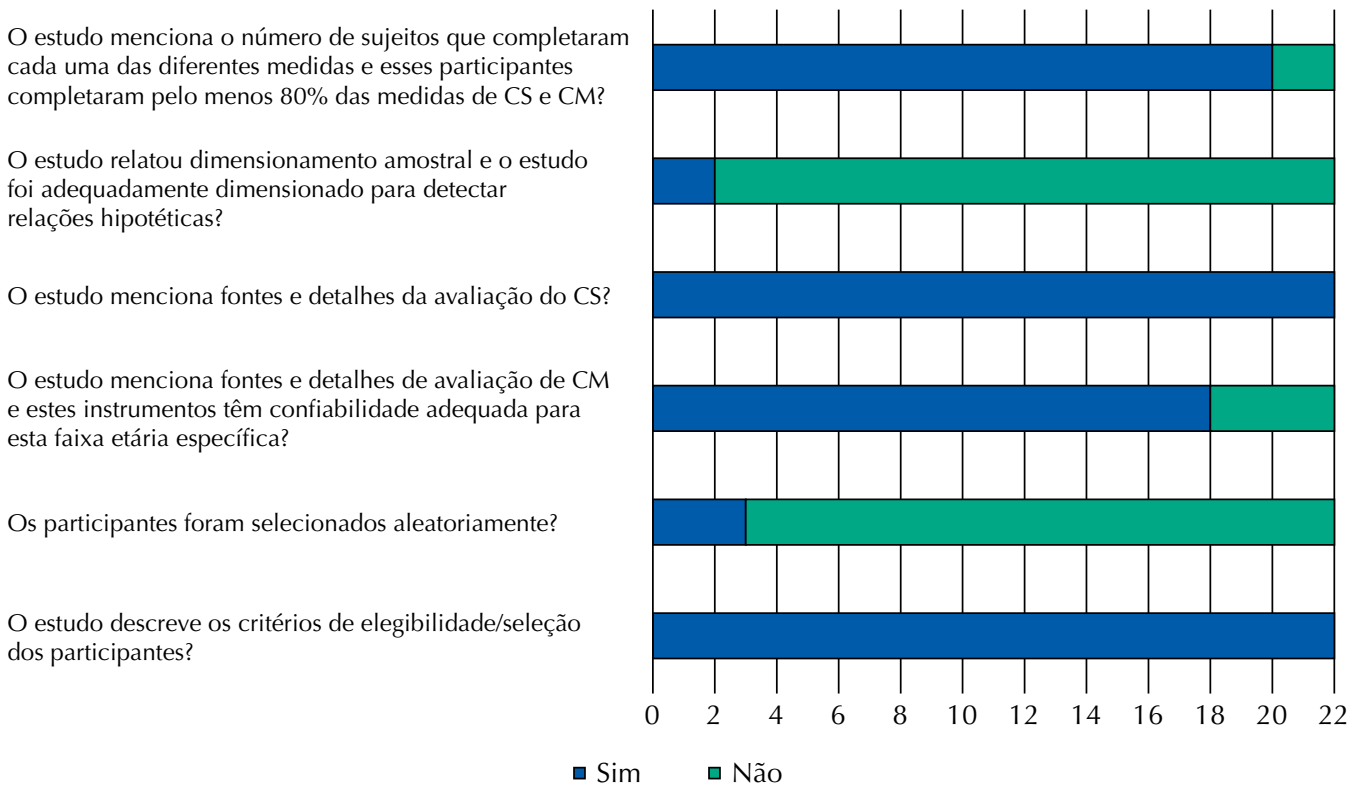

Figura 2. Análise por risco de viés dos artigos incluídos.

o outro ${ }^{48}$ encontrou associação incerta. Considerando os estudos longitudinais $(n=9)$, dois estudos de baixo risco de viés encontraram associação negativa entre CS e $\mathrm{CM}^{44,49}$, assim como outros cinco estudos de médio risco de viés ${ }^{30,40,43,45,52}$ encontraram associação negativa entre CS e CM. Em contraste, um estudo de alto risco de viés ${ }^{51}$ não encontrou associação e um estudo de moderado risco de viés ${ }^{47}$ encontrou associação incerta. Considerando os estudos transversais $(\mathrm{n}=11)$ um estudo de baixo risco de viés encontrou associação negativa entre CS e $\mathrm{CM}^{12}$ e quatro estudos de médio risco de viés ${ }^{33,37,46,53}$ encontraram associação negativa entre CS e CM. Em contraste, um estudo de baixo risco de viés ${ }^{39}$ e quatro estudos de médio risco de viés ${ }^{17,32,38,50}$ não encontraram associação, e um estudo de médio risco de viés ${ }^{42}$ encontrou associação incerta. 
Tabela 3. Distribuição dos estudos que investigaram comportamento sedentário e competência motora pelo risco de viés dentro dos estudos e pelo nível de evidência científica.

\begin{tabular}{|c|c|c|c|c|}
\hline Escolaridade & Estudos que demonstraram associação & $\begin{array}{l}\text { Estudos por risco } \\
\text { de viés }\end{array}$ & $\begin{array}{l}\text { Associação ou não associação de } \\
\text { acordo com o risco de viés }\end{array}$ & Nível de evidência \\
\hline Pré-escola $(\mathrm{n}=11)$ & $\begin{array}{c}\text { Associação negativa: } 5(45,4 \%) \\
\text { Não associação: } 5(45,4 \%) \\
\text { Associação incerta: } 1(9,1 \%)\end{array}$ & $\begin{array}{c}\text { Baixo: } 3(27,2 \%) \\
\text { Médio: } 7(63,6 \%) \\
\text { Alto: } 1(9,0 \%)\end{array}$ & $\begin{array}{c}\text { BRV: N:44,49; NA: }{ }^{39} \\
\text { MRV: } N: 40,43,53 ; \text { NA: }:^{32,50,17} ; ?:{ }^{42} \\
\text { ARV: NA: }{ }^{51}\end{array}$ & Evidência incerta \\
\hline $\begin{array}{l}\text { Ensino fundamental } \\
(\mathrm{n}=11)\end{array}$ & $\begin{array}{c}\text { Associação negativa: } 8(88,8 \%) \\
\text { Não associação: } 1(9,0 \%) \\
\text { Associação incerta: } 2(18,1)\end{array}$ & $\begin{array}{c}\text { Baixo: } 1(9,0 \%) \\
\text { Médio: } 10(90,9 \%)\end{array}$ & $\begin{array}{c}\text { BRV: } N:^{12} \\
\text { MRV: } N: 41,30,45,46,33,52,37\end{array}$ NA:38; ?:47,49 & $\begin{array}{c}\text { Evidência de } \\
\text { associação negativa }\end{array}$ \\
\hline
\end{tabular}

BRV: baixo risco de viés; MRV: médio risco de viés; ARV: alto risco de viés; N: associação negativa; NA: associação nula; ?: associação incerta.

Analisando a faixa etária de pré-escolares $(\mathrm{n}=11)$, dois estudos de baixo risco de viés ${ }^{44,49} \mathrm{e}$ três estudos de médio risco de viés ${ }^{40,43,53}$ encontraram associação negativa entre CS e CM. Em contraste, um estudo de baixo risco de viés ${ }^{39}$, três estudos de médio risco de viés ${ }^{17,32,50}$ e um estudo de alto risco de viés ${ }^{51}$ não encontraram associação, enquanto um estudo encontrou associação incerta ${ }^{42}$. Sendo assim, de acordo com os critérios estabelecidos, os resultados demonstram evidência incerta de associação entre CS e CM nessa faixa etária. Considerando a faixa etária de ensino fundamental $(\mathrm{n}=11)$, um estudo de baixo risco de viés $^{12}$ e sete estudos ${ }^{30,33,37,41,45,46,52}$ de médio risco de viés encontraram associação negativa entre CS e CM. Em contraste, um estudo de médio risco de viés ${ }^{38}$ não encontrou associação e dois estudos encontraram associação incerta. Portanto, os resultados indicam evidências de associação negativa entre CS e CM nesta faixa etária (Tabela 3).

Em suma, considerando o resultado das associações e o risco de viés, houve evidência científica para a associação negativa entre CS e CM de crianças e adolescentes do ensino fundamental e evidência científica incerta para associação em crianças pré-escolares.

\section{DISCUSSÃO}

A presente revisão sintetizou os resultados dos estudos que avaliaram associações entre CS e CM na infância e adolescência. De acordo com os resultados, pode-se afirmar que há evidência para associação negativa entre CS e CM nos anos do ensino fundamental. Mesmo que a maioria dos estudos examinados tenha usado um delineamento transversal, dificultando inferências sobre causalidade, sete estudos longitudinais apontaram associações negativas entre CS e CM, sugerindo que o tempo em CS pode prejudicar o desenvolvimento da $\mathrm{CM}^{30,40,43-45,49,52}$.

A metanálise de Engel et al..$^{54}$ sugere que a CM e os níveis de AF podem aumentar por meio de intervenções na infância, corroborando os achados de uma revisão prévia ${ }^{55}$, na qual as habilidades de controle de objetos foram fortemente associadas à AF em meninos, e as habilidades de locomoção foram associadas à AF em meninas. Um recente estudo longitudinal demonstrou a diminuição de AF e aumento do CS entre 6 e 11 anos $^{56}$. Uma hipótese plausível, baseada nos resultados encontrados no presente estudo e no modelo conceitual proposto por Stodden et al. ${ }^{24}$, é que a CM adequada poderia influenciar o aumento da AF e a diminuição do CS. É possível que crianças com excesso de CS tenham poucas oportunidades de desenvolvimento da $\mathrm{CM}^{30}$, aumentando ainda mais a probabilidade de adoção de CS (atividades de tela) nos momentos de lazer ${ }^{18,29}$ e gerando um ciclo comportamental vicioso com hábitos de vida não saudáveis associados ao surgimento de doenças crônico-degenerativas ${ }^{24}$. Assim, é importante que políticas públicas de saúde considerem a relação recíproca e dinâmica entre CS e CM e promovam, simultaneamente, a melhora da CM e a diminuição do CS, particularmente do tempo de lazer em atividades de tela durante a infância e adolescência.

Quanto aos fatores ambientais, o modelo teórico de Hulteen et al. ${ }^{57}$ sugere que as habilidades motoras variam em importância e popularidade de acordo com o contexto 
cultural e geográfico, com papel importante para a manutenção da AF ao longo da vida. Considerando o papel da atividade física como forma de intervenção para diminuir o CS e contribuir para o desenvolvimento da CM, é possível sugerir que quanto maior a CM em diferentes habilidades (por exemplo, chutar, arremessar, quicar e saltar), maior a gama de possibilidades de prática de AF que poderiam substituir o CS. O desenvolvimento da CM em habilidades que predominam no contexto cultural e geográfico em questão pode ser uma estratégia eficaz para substituir o CS por uma AF que a criança ou adolescente tenha competência para executar. Reforçando essa perspectiva, dados de um estudo finlandês ${ }^{41}$ apontam associação negativa entre a habilidade do chute e o CS, sendo o futebol um dos esportes juvenis predominantes no país ${ }^{58}$.

Os dados desta revisão reforçam a importância de políticas públicas integradas de educação e saúde focadas, simultaneamente, na melhoria da CM e diminuição do CS, particularmente do tempo de tela no lazer. Intervenções em crianças que passam tempo excessivo em CS devem incluir o desenvolvimento da CM de forma contínua, ou seja, deve-se levar em consideração a qualidade da prática de $\mathrm{AF}$, não apenas a quantidade de tempo dispendida nessa prática ${ }^{59,60}$. A melhora da CM pode ser uma estratégia promissora para reduzir o CS e aumentar a AF moderada a vigorosa, principalmente para as crianças com baixa $\mathrm{CM}^{12}$. É importante que futuras pesquisas sejam fortalecidas por avaliações longitudinais para proporcionar melhor compreensão da causalidade entre CS e CM.

Além disso, existe vasta margem a ser explorada, levando em consideração fatores ambientais, gênero e métodos de avaliação, bem como a lacuna existente na transição entre faixas etárias (crianças, adolescentes, adultos jovens e idosos). É plausível que a direção causal entre as variáveis seja influenciada pela faixa etária. Recomendam-se para futuros estudos a utilização de dois ou mais testes motores ${ }^{22,61} \mathrm{e}$ o uso do acelerômetro conjuntamente ao questionário para o melhor entendimento do CS e fatores ambientais. Sugerem-se também a seleção randomizada dos participantes e descrição do poder estatístico do estudo. Por fim, também é importante destacar que os resultados encontrados se limitam aos adolescentes até 14 anos, proporcionando vasto campo de pesquisa entre adolescentes e jovens adultos nesta temática.

O presente estudo contém algumas limitações. Embora a busca tenha sido abrangente, apenas estudos na língua inglesa foram incluídos. O viés relacionado ao relato seletivo das associações nos estudos pode ser uma possibilidade, e não foram considerados os ajustes nas associações entre CS e CM em função da prática de AF. Além disso, os resultados desta revisão são influenciados pelas limitações na base de evidências. Os efeitos negativos do CS podem ser compensados parcialmente pela prática de AF moderada a vigorosa (AFMV) ${ }^{31,62}$, sendo comportamentos independentes, ou seja, altos níveis de CS não necessariamente implicam em baixos níveis de AFMV e vice-versa; pessoas que atendem às recomendações de AFMV ( $\geq$ lh diária) ainda podem ter CS em muitas horas do dia ${ }^{63}$. Na realidade, até o momento, as recomendações de CS são restritas ao tempo de tela ${ }^{1,15,64}$. Do ponto de vista matemático, se for aplicada a recomendação de no mínimo três horas diárias de $\mathrm{AF}$ em qualquer intensidade (incluindo $1 \mathrm{~h}$ de AFMV) ${ }^{1,15}$, e considerando um período de $12 \mathrm{~h}$ de vigília, o tempo limite de CS seria de 9 h diárias. Portanto, é necessário um maior entendimento do contexto em que o CS se manifesta e sua associação com o desenvolvimento global da criança ou adolescente.

Outra limitação é a heterogeneidade nas formas utilizadas para avaliar o $\mathrm{CS}^{65}$ (questionários com informações sobre o tempo total de tela ou somente tempo de TV/computador ou tempo sentado e medidas diretas de acelerometria), impossibilitando uma diferenciação do CS em atividades de tela/lazer e do CS em outros domínios, como tempo sentado no deslocamento ou nos estudos. O uso de diferentes testes motores para aferir a CM também é uma limitação importante. No total, pelo menos 10 avaliações diferentes de CM foram utilizadas. Na literatura a CM pode ser avaliada por meio de medidas orientadas ao processo ou ao produto do movimento, e isso pode influenciar a magnitude das associações ${ }^{22}$. 
Finalmente, os resultados desta revisão sistemática demonstram que há evidências de associação negativa entre CS e CM em crianças e adolescentes no período do ensino fundamental, embora a evidência seja incerta nos anos pré-escolares. Os fatores ambientais, assim como o método de avaliação e faixa etária, podem ser determinantes para a melhor compreensão do fenômeno investigado. Para o melhor entendimento das associações entre CS e CM, sugerimos o estabelecimento de critérios padronizados para a condução dos estudos, destacando o contexto em que o CS se manifesta (por exemplo, em situações de lazer ou atividades escolares). Melhorar a CM predominante no contexto sociocultural em que a pessoa está inserida pode contribuir para a diminuição do CS e promover o engajamento em um estilo de vida ativo em longo prazo para as crianças e adolescentes.

\section{REFERÊNCIAS}

1. Tremblay MS, LeBlanc AG, Kho ME, Saunders TJ, Larouche R, Colley RC, et al. Systematic review of sedentary behaviour and health indicators in school-aged children and youth. Int J Behav Nutr Phys Act. 2011;8:98. https://doi.org/10.1186/1479-5868-8-98

2. World Health Organization. Global status report on noncommunicable diseases 2010. Geneva $(\mathrm{CH})$ : WHO; 2011.

3. Tremblay MS, Aubert S, Barnes JD, Saunders TJ, Carson V, Latimer-Cheung AE et al. Sedentary Behavior Research Network (SBRN) - Terminology Consensus Project process and outcome. Int J Behav Nutr Phys Act. 2017;14(1):75. https://doi.org/10.1186/s12966-017-0525-8

4. Jones RA, Hinkley T, Okely AD, Salmon J. Tracking physical activity and sedentary behavior in childhood: a systematic review. Am J Prev Med. 2013;44(6):651-8. https://doi.org/10.1016/j.amepre.2013.03.001

5. Gordon-Larsen P, Nelson MC, Popkin BC. Longitudinal physical activity and sedentary behavior trends: adolescence to adulthood. Am J Prev Med. 2004;27(4):277-83. https://doi.og/ 10.1089/chi.2013.0021

6. Kohl HW 3rd, Craig CL, Lambert EV, Inoue S, Alkandari JR, Leetongin G, et al. The pandemic of physical inactivity: global action for public health. Lancet. TV viewing and physical activity are independently associated with metabolic risk in children: The European Youth Heart Study. 2012;380(9838):294-305. https://doi.org/10.1016/s0140-6736(12)60898-8

7. Ekelund U, Brage S, Froberg K, Harro M, Anderssen SA, Sardinha LB, et al. TV viewing and physical activity are independently associated with metabolic risk in children: The European Youth Heart Study. PLoS Med. 2006;3(12):e488. https://doi.org/10.1371/journal.pmed.0030488

8. Mark AE, Janssen I. Relationship between screen time and metabolic syndrome in adolescents. J Public Health (Oxf). 2008;30(2):153-60. https://doi.org/10.1093/pubmed/fdn022

9. Salmon J, Dunstan D, Owen N. Should we be concerned about children spending extended periods of time in sedentary pursuits even among the highly active? Int J Pediatr Obes. 2008;3(2):66-8. https://doi.org/10.1080/17477160802169672

10. Pate RR, Mitchell JA, Byun W, Dowda M. Sedentary behaviour in youth. Br J Sports Med. 2011;45(11): 906-13. https://doi.org/10.1136/bjsports-2011-090192

11. Bibiloni MM, Pich J, Córdova A, Pons A, Tur JA. Association between sedentary behaviour and socioeconomic factors, diet and lifestyle among the Balearic Islands adolescents. BMC Public Health. 2012;12:718. https://doi.org/10.1186/1471-2458-12-718

12. Adank AM, Van Kann DHH, Hoeboer JJAA, Vries SI, Kremer SPJ, Vos SB. Investigating motor competence in association with sedentary behavior and physical activity in 7- to 11-year-old children. Int J Environ Res Public Health. 2018;15(11):2470. https://doi.org/10.3390/ijerph15112470

13. Biddle SJH, Marshall SJ, Gorely T, Cameron N. Temporal and environmental patterns of sedentary and active behaviors during adolescents' leisure time. Int J Behav Med. 2009;16(3):278-86. https://doi.org/10.1007/s12529-008-9028-y

14. Mathers M, Canterford L, Olds T, Hesketh K, Ridley K, Wake M. Electronic media use and adolescent health and well-being: cross-sectional community study. Acad Pediatr. 2009;9(5):307-14. https://doi.org/10.1016/j.acap.2009.04.003

15. World Health Organization. Guidelines on physical activity, sedentary behaviour and sleep for children under 5 years of age. Geneva $(\mathrm{CH})$ : WHO; 2019. 
16. Tremblay MS, Carson V, Chaput JP, Gorber SC, Dinh T, Duggan M, et al. Canadian 24-hour movement guidelines for children and youth: an integration of physical activity, sedentary behaviour, and sleep. Appl Physiol Nutr Metab. 2016;41(6 Suppl 3):S311-27. https://doi.org/10.1139/apnm-2016-0151

17. Webster EK, Martin CK, Staiano AE. Fundamental motor skills, screen-time, and physical activity in preschoolers. J Sport Health Sci. 2019;8(2):114-21. https://doi.org/10.1016/j.jshs.2018.11.006

18. Andrade Neto F, Eto FN, Pereira TSS, Carletti L, Molina MCB. Active and sedentary behaviours in children aged 7 to 10 years old: the urban and rural contexts, Brazil. BMC Public Health. 2014;14:1174. https://doi.org/10.1186/1471-2458-14-1174

19. Moore SA, Faulkner G, Rhodes RE, Brussoni M, Chulak-Bozzer T, Ferguson LJ, et al. Impact of the COVID-19 virus outbreak on movement and play behaviours of Canadian children and youth a national survey. Int J Behav Nutr Phys Act. 2020;17:85. https://doi.org/10.1186/s12966-020-00987-8

20. Brian A, Pennell A, Taunton S, Starrett A, Howard-Shaughnessy C, Goodway JD, et al. Motor competence levels and developmental delay in early childhood: a multicenter cross-sectional study conducted in the USA. Sports Med. 2019;49(10):1609-18. https://doi.org/10.1007/s40279-019-01150-5

21. Valentini NC, Clark JE, Whitall J. Developmental co-ordination disorder in socially disadvantaged Brazilian children. Child Care Health Dev. 2015;41(6):9709. https://doi.org/10.1111/cch.12219

22. Ré AHN, Logan SW, Cattuzzo MT, Henrique RS, Tudela MC, Stodden DF. Comparison of motor competence levels on two assessments across childhood. J Sports Sci. 2018;36(1):1-6. https://doi.org/10.1080/02640414.2016.1276294

23. Robinson LE, Stodden DF, Barnett LM, Lopes VP, Logan SW, Rodrigues LP, et al. Motor competence and its effect on positive developmental trajectories of health. Sports Med. 2015;45(9):1273-84. https://doi.org/10.1007/s40279-015-0351-6.3

24. Stodden DF, Goodway JD, Langendorfer SJ, Roberton MA, Rudisill ME, Garcia $\mathrm{C}$, et al. A developmental perspective on the role of motor skill competence in physical activity: an emergent relationship. Quest. 2008;60(2):290-306. https://doi.org/10.1080/00336297.2008.10483582

25. Henrique RS, Ré AHN, Stodden DF, Fransen J, Campos CMC, Queiroz DR, et al. Association between sports participation, motor competence and weight status: a longitudinal study. J Sci Med Sport. 2016;19(10):825-9. https://doi.org/10.1016/j.jsams.2015.12.512

26. Lima RA, Pfeiffer K, Larsen LR, Bugge A, Moller NC, Anderson LB, Stodden DF. Physical activity and motor competence present a positive reciprocal longitudinal relationship across childhood and early adolescence. J Phys Act Health. 2017;14(6):440-7. https://doi.org/10.1123/jpah.2016-0473

27. Lubans DR, Morgan PJ, Cliff DP, Barnett LM, Okely AD. Fundamental movement skills in children and adolescents: review of associated health benefits. Sports Med. 2010;40(12):1019-35. https://doi.org/10.2165/11536850-000000000-00000

28. Cattuzzo MT, Henrique RS, Ré AHN, Oliveira IS, Melo BM, Moura M, et al. Motor competence and health related physical fitness in youth: a systematic review. J Sci Med Sport. 2016;19(2):123-9. https://doi.org/10.1016/j.jsams.2014.12.004

29. Hardy LL, Ding D, Peralta LR, Mihrshahi S, Merom D. Association between sitting, screen time, fitness domains, and fundamental motor skills in children aged 5-16 years: cross-sectional population study. J Phys Act Health. 2018;15(12):933-40. https://doi.org/10.1123/jpah.2017-0620

30. Drenowatz C, Greier K. Cross-sectional and longitudinal association of sports participation, media consumption and motor competence in youth. Scand J Med Sci Sports. 2019;29(6):854-61. https://doi.org/10.1111/sms.13400

31. Cliff DP, Hesketh KD, Vella SA, Hinkley T, Tsiros MD, Ridgers ND, et al. Objectively measured sedentary behaviour and health and development in children and adolescents: systematic review and meta-analysis. Obes Rev. 2016;17(4):330-44. https://doi.org/10.1111/obr.12371

32. Cliff DP, Okely AD, Smith LM, McKeen K. Relationships between fundamental movement skills and objectively measured physical activity in preschool children. Pediatr Exerc Sci. 2009;21(4):436-49. https://doi.org/10.1123/pes.21.4.436 
33. Lopes L, Santos R, Pereira B, Lopes VP. Associations between sedentary behavior and motor coordination in children. Am J Hum Biol. 2012;24(6):746-52. https://doi.org/10.1002/ajhb.22310

34. Williams HG, Pfeiffer KA, O'Neill JR, Dowda M, Mclver KL, Brown WH, et al. Motor skill performance and physical activity in preschool children. Obesity (Silver Spring). 2008;16(6):1421-26. https://doi.org/10.1038/oby.2008.214

35. Moher D, Shamseer L, Clarke M, Ghersi D, Liberati A, Petticrew M, et al. Preferred reporting items for systematic review and meta-analysis protocols (PRISMA-P) 2015 statement. Syst Rev. 2015;4(1):1. https://doi.org/10.1186/2046-4053-4-1

36. Richardson WS, Wilson MC, Nishikawa J, Hayward RS. The well-built clinical question: a key to evidence-based decisions. ACP J Club. 1995;123(3):A12-3.

37. Wrotniak BH, Epstein LH, Dorn JM, Jones KE, Kondilis VA. The relationship between motor proficiency and physical activity in children. Pediatrics. 2006;118(6):e1758-65. https://doi.org/10.1542/peds.2006-0742

38. Burns RD, Kim Y, Byun W, Brusseau TA. Associations of school day sedentary behavior and physical activity with gross motor skills: use of compositional data analysis. J Phys Act Health. 2019;16(10):811-17. https://doi.org/10.1123/jpah.2018-0549

39. Barnett LM, Hinkley T, Okely AD, Hesketh K, Salmon J. Use of electronic games by young children and fundamental movement skills? Percept Mot Skills. 2012;114(3):1023-34. https://doi.org/10.2466/10.13.PMS.114.3.1023-1034

40. Cadoret G, Bigras N, Lemay L, Lehrer J, Lemire J. Relationship between screen-time and motor proficiency in children: a longitudinal study. Early Child Dev Care. 2018;188(2):231-9. https://doi.org/10.1080/03004430.2016.1211123

41. Capio CM, Sit CHP, Eguia KF, Abernethy B, Masters RSW. Fundamental movement skills training to promote physical activity in children with and without disability: a pilot study. J Sport Health Sci. 2015;4(3):235-43. https://doi.org/10.1016/j.jshs.2014.08.001

42. Famelia R, Tsuda E, Bakhtiar S, Goodway JD. Relationships among perceived and actual motor skill competence and physical activity in Indonesian preschoolers. J Mot Learn Dev. 2018;6 Suppl 2:S403-23. https://doi.org/10.1123/jmld.2016-0072

43. Gu X. Fundamental motor skill, physical activity, and sedentary behaviour in socioeconomically disadvantaged kindergarteners. Psychol Health Med. 2016;21(7):871-81. https://doi.org/10.1080/13548506.2015.1125007

44. Gu X, Keller MJ, Weiller-Abels KH, Zhang T. The roles of physical activity and sedentary behavior on Hispanic children's mental health: a motor skill perspective. Qual Life Res. 2018;27(7):185- 93. https://doi.org/10.1007/s11136-017-1687-1

45. Gu X, Chen S, Zhang X. Young Hispanic and non-Hispanic children's fundamental motor competence and physical activity behaviors. J Mot Learn Dev. 2019;7(2):180-93. https://doi.org/10.1123/jmld.2018-0003

46. Jaakkola T, Kalaja S, Liukkonen J, Jutila A, Virtanen P, Watt A. Relations among physical activity patterns, lifestyle activities, and fundamental movement skills for Finnish students in grade 7. Percept Mot Skills. 2009;108(1):97-111. https://doi.org/10.2466/pms.108.1.97-111

47. Jaakkola T, Hakonen H, Kankaanpää A, Joensuu L, Kulmala J, Kallio J, et al. Longitudinal associations of fundamental movement skills with objectively measured physical activity and sedentariness during school transition from primary to lower secondary school. J Sci Med Sport. 2019;22(1):85-90. https://doi.org/10.1016/j.jsams.2018.07.012

48. Laukkanen A, Pesola A, Havu M, Sääkslahti A, Finni T. Relationship between habitual physical activity and gross motor skills is multifaceted in 5- to 8-year-old children. Scand J Med Sci Sports. 2014;24(2):e102-10. https://doi.org/10.1111/sms.12116

49. Lloyd M, Saunders TJ, Bremer E, Tremblay MS. Long-term importance of fundamental motor skills: a 20-year follow-up study. Adapt Phys Act Q. 2014;31(1):67-78. https://doi.org/10.1123/apaq.2013-0048

50. Lopes $\mathrm{V}$, Barnett L, Rodrigues L. Is there an association among actual motor competence, perceived motor competence, physical activity, and sedentary behavior in preschool children? J Mot Learn Dev. 2016;4(2):129-41. https://doi.org/10.1123/jmld.2015-0012

51. Matarma T, Tammelin T, Kulmala J, Koski P, Hurme S, Lagström H. Factors associated with objectively measured physical activity and sedentary time of 5-6-year-old children in the STEPS Study. Early Child Dev Care. 2017;187(12):1863-73. https://doi.org/10.1080/03004430.2016.1193016 
52. Smith L, Fisher A, Hamer M. Prospective association between objective measures of childhood motor coordination and sedentary behaviour in adolescence and adulthood. Int J Behav Nutr Phys Act. 2015;12:75. https://doi.org/10.1186/s12966-015-0236-y

53. Tsuda E, Goodway JD, Famelia R, Brian A. Relationship between fundamental motor skill competence, perceived physical competence and free-play physical activity in children. Res Q Exerc Sport. 2020;91(1):55-63. https://doi.org/10.1080/02701367.2019.1646851

54. Engel AC, Broderick CR, Doorn N, Hardy LL, Parmenter BJ. Exploring the relationship between fundamental motor skill interventions and physical activity levels in children: a systematic review and meta-analysis. Sports Med. 2018;48(8):1845-57. https://doi.org/10.1007/s40279-018-0923-3

55. Logan SW, Webster EK, Getchell N, Pfeiffer KA, Robinson LE. Relationship between fundamental motor skill competence and physical activity during childhood and adolescence: a systematic review. Kinesiol Rev. 2015;4(4):416-26. https://doi.org/10.1123/kr.2013-0012

56. Schwarzfischer P, Gruszfeld D, Stolarczyk A, Ferre N, Escribano J, Rousseaux D, et al. Physical activity and sedentary behavior from 6 to 11 years. Pediatrics. 2019;143(1):e20180994. https://doi.org/10.1542/peds.2018-0994

57. Hulteen RM, Morgan PJ, Barnett LM, Stodden DF, Lubans DR. Development of foundational movement skills: A conceptual model for physical activity across the lifespan. Sports Med. 2018;48(7):1533-40. https://doi.org/10.1007/s40279-018-0892-6

58. Kokko S, Kannas L, Villberg J. Health promotion profile of youth sports clubs in Finland: club officials' and coaches' perceptions. Health Promot Int. 2009;24(1):26-35. https://doi.org/10.1093/heapro/dan040

59. Ribeiro EHC, Guerra PH, Oliveira AC, Silva KS, Santos P, Santos R, et al. Latin American interventions in children and adolescents' sedentary behavior: a systematic review. Rev Saude Publica. 2020;54:59. https://doi.org/10.11606/s1518-8787.2020054001977

60. Ré AHN, Okely AD, Logan SW, Silva MMLM, Cattuzzo MT, Stodden DF. Relationship between meeting physical activity guidelines and motor competence among low-income school youth. J Sci Med Sport. 2020;23(6):591-5. https://doi.org/10.1016/j.jsams.2019.12.014

61. Logan SW, Barnett LM, Goodway JD, Stodden DF. Comparison of performance on processand product-oriented assessments of fundamental motor skills across childhood. J Sports Sci. 2017;35(7):634-41. https://doi.org/10.1080/02640414.2016.1183803

62. Ekelund U, Steene-Johannessen J, Brown WJ, Fagerland MW, Owen N, Powell KE, et al. Does physical activity attenuate, or even eliminate, the detrimental association of sitting time with mortality? A harmonised meta- analysis of data from more than 1 million men and women. Lancet. 2016;388(10051):1302-10. https://doi.org/10.1016/S0140-6736(16)30370-1

63. Verloigne M, Van Lippevelde W, Maes L, Yildirim M, Chinapaw M, Manios Y, et al. Levels of physical activity and sedentary time among 10- to 12-year-old boys and girls across 5 European countries using accelerometers: an observational study within the ENERGY-project. Int J Behav Nutr Phys Act. 2012;9:34. https://doi.org/10.1186/1479-5868-9-34

64. Okely AD, Gersi D, Hesketh KD, Santos R, Loughran SP, Cliff DP, et al. A collaborative approach to adopting/adapting guidelines -The Australian 24-Hour Movement Guidelines for the early years (Birth to 5 years): an integration of physical activity, sedentary behavior, and sleep. BMC Public Health. 2017;17:869. https://doi.org/10.1186/s12889-017-4867-6

65. Strain T, Milton K, Dall P, Standage M, Mutrie N. How are we measuring physical activity and sedentary behaviour in the four home nations of the UK? A narrative review of current surveillance measures and future directions. Br J Sports Med. 2019;54(21):1269-76. https://doi.org/10.1136/bjsports-2018-100355

Financiamento: O presente trabalho foi realizado com apoio da Coordenação de Aperfeiçoamento de Pessoal de Nível Superior - Brasil (CAPES) - Código de Financiamento 001, auxílios da FAPESP (processos 2017/08496-6 e 2020/15646-7) e do Programa Unificado de Bolsas da Universidade de São Paulo.

Contribuição dos Autores: Concepção e planejamento do estudo: GS, PHG, AHNR. Coleta de dados: GS, SAM. Análise e interpretação de dados; GS, PHG, SAM, AHNR. Preparação e redação do manuscrito; GS, PHG, SAM, AHNR. Revisão crítica do manuscrito: GS, PHG, SAM, ABDS, MTC, AHNR. Aprovação final: GS, PHG, SAM, ABDS, MTC, AHNR.

Conflito de Interesses: Os autores declaram não haver conflito de interesses. 